José Arturo Sosa Echeverría

El nuevo mecanismo para la integración de los congresos con diputados de representación proporcional, una fórmula más democrática Revista Xihmai XIII (26), 51-78, julio-diciembre 2018

\title{
Xíhmai
}

Universidad La Salle Pachuca

xihmai@lasallep.edu.mx

Teléfono: 01(771) 7170213 ext. 1406

Fax: 01(771) 7170309

ISSN (versión impresa):1870-6703

México

José Arturo Sosa Echeverría

EL NUEVO MECANISMO PARA LA INTEGRACIÓN DE LOS CONGRESOS CON DIPUTADOS DE REPRESENTACIÓN PROPORCIONAL, UNA FÓRMULA MÁS DEMOCRÁTICA

THE NEW MECHANISM FOR THE INTEGRATION OF CONGRESSES WITH MEMBERS OF PROPORTIONAL REPRESENTATION, A MORE DEMOCRATIC FORMULA

Xihmai, año 2018/vol. XIII, número 26

Universidad La Salle Pachuca

pp. $51-78$ 
José Arturo Sosa Echeverría

El nuevo mecanismo para la integración de los congresos con diputados de representación proporcional, una fórmula más democrática Revista Xihmai XIII (26), 51-78, julio-diciembre 2018

\section{Xihmai 52}


José Arturo Sosa Echeverría

El nuevo mecanismo para la integración de los congresos con diputados de representación proporcional, una fórmula más democrática

Revista Xihmai XIII (26), 51-78, julio-diciembre 2018

\title{
EL NUEVO MECANISMO PARA LA INTEGRACIÓN DE LOS CONGRESOS CON DIPUTADOS DE REPRESENTACIÓN PROPORCIONAL, UNA FÓRMULA MÁS DEMOCRÁTICA
}

\author{
THE NEW MECHANISM FOR THE INTEGRATION OF CONGRESSES WITH \\ MEMBERS OF PROPORTIONAL REPRESENTATION, A MORE DEMOCRATIC \\ FORMULA
}

José Arturo Sosa Echeverría

Licenciado en Derecho por la Universidad Autónoma del Estado de Hidalgo; abogado litigante; colaborador en el Instituto Estatal Electoral de Hidalgo con los cargos de Director Ejecutivo de Capacitación Electoral y Educación Cívica y Director Ejecutivo Jurídico hasta 2015; Subprocurador de Asuntos Electorales a partir del 1 de diciembre de 2015 a la fecha.

Docente en el nivel medio superior en las asignaturas de Derecho Mercantil e Introducción al Estudio del Derecho; en el nivel universitario con las asignaturas de Derecho Civil y Derecho Electoral; profesor de la Facultad de Derecho de la Universidad La Salle Pachuca en las asignaturas de Derecho Electoral, Derecho Procesal Electoral y Derecho Procesal Penal. Actualmente, trabaja en la Procuraduría General de Justicia del Estado de Hidalgo. arturososae@yahoo.com.mx

\section{Recibido 10-02-18 Corregido 20-03-18 Aceptado 20-05-18}

\section{Resumen}

Desde la inclusión del sistema electoral mixto para la composición de los órganos legislativos del país, solo existía un mecanismo para acceder a las curules de representación proporcional a través de las listas que presentaban los partidos políticos para esa elección en particular; de manera muy reciente, algunas legislaturas locales han adoptado un mecanismo que incluye al sistema tradicional y un nuevo método de acceder a esos espacios: además de esa lista tradicional se prevé la conformación de la segunda lista, compuesta por los candidatos que participaron en la elección bajo el principio de mayoría relativa; ambas listas la tomará el órgano electoral respectivo para que de forma alternada se asignen los diputados que a cada partido correspondan. Esto implica que, aunque hayan 
José Arturo Sosa Echeverría

El nuevo mecanismo para la integración de los congresos con diputados de representación proporcional, una fórmula más democrática

Revista Xihmai XIII (26), 51-78, julio-diciembre 2018

perdido la elección, los candidatos que hicieron campaña y recibieron el voto directo del electorado tienen posibilidad de arribar al Congreso, lo que genera un sentido legitimador en los diputados plurinominales porque son ellos a quienes la ciudadanía otorgó su voto de manera directa, además que, de este modo, se fomenta la participación ciudadana porque el elector ve reflejado su voto en la persona a quien él se lo otorgó.

Palabras clave: Democracia, Elección, Representación Proporcional.

\begin{abstract}
Since the inclusion of the mixed electoral system for the composition of the legislative bodies of the country, there was only a mechanism to access the proportional representation seats, this was, by presenting lists of political parties for this election in particular; Very recently, some local legislatures have adopted a mechanism that includes the traditional system and a new method of accessing these spaces, this in addition to the traditional list today provides for the formation of the second list consisting of candidates who participated in the election under the principle of relative majority; both lists will be made by the respective electoral body to go alternately assigning deputies that correspond to each party. This means that candidates who campaigned and received the direct vote of the electorate, but lost the election, have a chance arriving to Congress, creating a legitimizing sense in the multi-named deputies, because they, whom the citizenship granted their vote directly, besides that it encourages citizen participation because the elector his vote reflected the person to whom he gave it.
\end{abstract}

Keywords: Democracy, Election, Proportional Representation

\title{
Antecedentes \\ Diputados de Partido
}

El 22 de julio del año de 1963 fue presentado el decreto de adiciones y reformas al artículo 54 de la Constitución Política de los Estados Unidos Mexicanos por el que se crean los diputados de partido. El entonces Presidente de la República, Adolfo López Mateos, expuso los motivos de la iniciativa, entre los que destaco:

Es evidente el hecho de que no han podido encontrar acceso al Congreso de la Unión los diversos partidos políticos o las varias corrientes de opinión que actúan en la República; de ahí que, con frecuencia, se haya criticado al sistema mexicano de falta de flexibilidad para dar oportunidad a las minorías políticas, que se duelen de que un solo partido mayoritario obtenga la casi totalidad de los puestos de representación popular... Con esas bases se realiza la reforma, por la que se institucionaliza la

Xihmai 54 
José Arturo Sosa Echeverría

El nuevo mecanismo para la integración de los congresos con diputados de representación proporcional, una fórmula más democrática

Revista Xihmai XIII (26), 51-78, julio-diciembre 2018

representación minoritaria a través de la creación de los diputados de partido y se establece el procedimiento para acreditarlos ante las autoridades competentes.

El efecto de la incorporación de los diputados de partido al sistema político mexicano, en palabras del mandatario en turno Adolfo López Mateos, obedeció a la necesidad de contar, en los espacios del poder público (en este caso la Cámara de Diputados), con personajes provenientes de fuerzas políticas que representaban a las minorías en nuestro país.

Según la reforma, para que los partidos políticos contendientes en los procesos electorales de la época accedieran a la asignación de los diputados de partido debían de obtener el umbral mínimo del $2.5 \%$ de la votación, con lo que, de manera automática, se hacían merecedores a cinco curules, incrementándose un diputado más por cada medio punto porcentual que obtuvieran por arriba del mínimo señalado, hasta el máximo permitido equivalente a veinte diputados, incluyéndose en esta cifra a los que lograran obtener por el principio de mayoría relativa.

La primera elección en que se aplicó la reforma de 1963 fue la celebrada el 5 de julio de 1964, siendo Presidente de la República Gustavo Díaz Ordaz; los resultados que arrojaron los cómputos hicieron que el Partido Acción Nacional obtuviera veinte diputados de partido; el Partido Popular Socialista obtuviera nueve y el Partido Auténtico de la Revolución Mexicana cinco. Estas cifras se mantendrían casi inalteradas en las décadas posteriores, lo que significó una muy escasa presencia de las minorías en la Cámara de Diputados.

Los denominados diputados de partido son, así, el antecedente más remoto que tenemos en nuestro país en relación a lo que hoy se conoce como la representación proporcional.

\section{La Representación Proporcional}

Para la elección presidencial del año de 1976, se hizo evidente una crisis política en nuestro país, ya que hubo sólo un candidato registrado para contender a la Presidencia de la República en la persona de José López Portillo, lo que de suyo afectó la legitimidad del primer mandatario electo en aquella contienda y se hizo latente la problemática de la representatividad, además de que puso en tela de juicio el modelo democrático contemplado en la Constitución.

Con motivo de lo anterior, se hizo necesaria una reforma política que data del año 1977. Esta transformación implicó una importante serie de cambios legales gestados desde la Secretaría de Gobernación en la administración de López Portillo, encabezada por el político, intelectual e historiador Jesús Reyes Heroles. 
José Arturo Sosa Echeverría

El nuevo mecanismo para la integración de los congresos con diputados de representación proporcional, una fórmula más democrática

Revista Xihmai XIII (26), 51-78, julio-diciembre 2018

La reforma del 6 de diciembre de 1977 establece que la Cámara de Diputados se integrará con trescientos miembros mediante el sistema de distritos electorales uninominales y hasta cien diputados electos según el principio de representación proporcional mediante el sistema de listas regionales. La elección de curules bajo el principio de representación proporcional estaba sujeta a las siguientes bases constitucionales: 1) Para obtener el registro de las listas regionales, el partido político debía acreditar que participaba con candidatos a diputados por el principio de mayoría relativa en por lo menos la tercera parte de los trescientos distritos uninominales, es decir, debía registrarse por cada instituto político a por lo menos cien candidatos; 2) se podrían asignar curules por el principio de representación proporcional a los partidos que no hubieran obtenido sesenta o más constancias de mayoría y que obtuvieran el 1.5\% del total de la votación emitida para todas las listas regionales en las circunscripciones plurinominales; 3) la fórmula para la asignación de diputaciones por el principio de representación proporcional se hacía conforme a un cociente electoral; y 4) para el supuesto de que dos o más partidos obtuvieran en su conjunto noventa o más constancias de mayoría, solamente se repartía el 50\% de las curules que deban asignarse por el principio de representación proporcional.

La reforma del 15 de diciembre del año de 1986 incrementó de cien a doscientas el número de diputados electos por el principio de representación proporcional, mantuvo el umbral mínimo de votación para acceder a la asignación equivalente al $1.5 \%$ del total de la votación emitida para las listas regionales de las cinco circunscripciones y modificó el procedimiento para la asignación de estos espacios, dentro de los que destaco:

Si algún partido obtenía el $51 \%$ o más de la votación nacional efectiva y el número de sus constancias de mayoría relativa representaba un porcentaje del total de la Cámara, inferior a su referido porcentaje de votos, tenía derecho a la asignación hasta que la suma de diputados obtenida por ambos principios representara el mismo porcentaje de votos; b) Ningún partido tenía derecho a que le fueran reconocidos más de 350 diputados, que representaban el $70 \%$ de la integración total de la Cámara, aun cuando hubiere obtenido un porcentaje de votos superior; c) Si ningún partido obtenía el 51\% de la votación nacional efectiva y ninguno alcanzaba con sus constancias de mayoría relativa la mitad más uno de los miembros de la Cámara, al partido con más constancias de mayoría se le asignaban diputados hasta alcanzar la mayoría absoluta de la Cámara. En caso de empate en el número de constancias, la mayoría absoluta era asignada al partido que hubiera alcanzado la mayor votación a nivel nacional en la elección de diputados por mayoría relativa.

1990 también fue un año de reformas que alcanzaron a la representación proporcional, las adecuaciones importantes son: acreditar que se participaba con candidatos a diputados por mayoría relativa en por lo menos doscientos distritos Xihmai 56 
José Arturo Sosa Echeverría

El nuevo mecanismo para la integración de los congresos con diputados de representación proporcional, una fórmula más democrática

Revista Xihmai XIII (26), 51-78, julio-diciembre 2018

uninominales; si ningún partido político obtenía por lo menos el $35 \%$ de la votación nacional emitida, se les otorgaban diputados suficientes para que su representación en la Cámara, por ambos principios, correspondiera, en su caso, a su porcentaje de votos obtenidos; al partido político que obtuviera el mayor número de constancias de mayoría y el 35\% de la votación nacional, se le asignaban los diputados suficientes para alcanzar la mayoría absoluta de la Cámara. También se le asignaban dos diputados, adicionalmente a la mayoría absoluta, por cada $1 \%$ de la votación obtenida por encima del $35 \%$ y hasta menos del $60 \%$ y, al partido político que obtuviera entre el $60 \%$ y el $70 \%$ de la votación nacional y sus constancias de mayoría relativa representaran un porcentaje del total de la Cámara inferior a su porcentaje de votos, se le asignaban diputados hasta que la suma de diputados obtenidos por ambos principios representara el mismo porcentaje de votos.

La reforma del año de 1993 modificó mínimamente la asignación de la representación proporcional, destacándose que en ningún caso un partido político podría contar con más de trescientos quince diputados por ambos principios; ningún partido político que hubiera obtenido el $60 \%$ o menos de la votación nacional emitida podía contar con más de trescientos diputados por ambos principios; si un partido político obtenía más del $60 \%$ de la votación nacional emitida, se le asignaban diputados hasta que el número de diputados por ambos principios fuera igual a su porcentaje de votación nacional emitida sin rebasar el límite de trescientos quince. Las diputaciones que restaban después de asignar las que le correspondían a un partido político que se ubicara en los dos supuestos anteriores, se otorgaba a los demás partidos con derecho a ello en proporción directa con sus respectivas votaciones nacionales.

Por último, la reforma de 1996 que se mantiene hasta esta fecha mantiene el requisito para participar en la asignación de los doscientos diputados por el principio de representación proporcional si se ha participado en, al menos, en el mismo número de distritos uninominales; alcanzar por lo menos el 3\% de la votación válida emitida para las listas regionales de las circunscripciones plurinominales; ningún partido político podrá contar con más de trescientos diputados por ambos principios; establece el límite a la sobre representación anunciando que ningún partido político podrá contar con un número de diputados por ambos principios que representen un porcentaje del total de la Cámara que exceda en ocho puntos a su porcentaje de votación nacional emitida; las curules se adjudicarán en proporción directa con las respectivas votaciones nacionales efectivas. 
José Arturo Sosa Echeverría

El nuevo mecanismo para la integración de los congresos con diputados

de representación proporcional, una fórmula más democrática

Revista Xihmai XIII (26), 51-78, julio-diciembre 2018

\section{Representación proporcional}

Se denomina representación proporcional,

en el ámbito político-electoral, a la relación directamente proporcional entre el número de votos emitidos por los electores y la distribución de los cargos electivos o los escaños parlamentarios entre los partidos políticos contendientes en un proceso eleccionario. Los sistemas electorales que la establecen persiguen que el número de representantes elegidos guarde proporción con el número de votos depositados por los electores (Borja).

La Academia de la Lengua Española define a la representación proporcional como el "procedimiento electoral que establece una proporción entre el número de votos obtenidos por cada partido o tendencia y el número de sus representantes elegidos" (1992).

La representación proporcional, entendida a la luz del sistema electoral mexicano, la podemos definir como una forma de acceder a los órganos de gobierno colegiados, electos popularmente, y que se obtienen en proporción directa a los porcentajes de votación alcanzados en la elección correspondiente, siempre y cuando se hayan cumplido con los requisitos legales conducentes.

En otras palabras, y refiriéndonos al caso del sistema electoral de nuestro país, los representantes del pueblo electos mediante el sufragio deberán corresponder en porcentaje total del órgano elegido con el porcentaje de votos recibido por el partido que los postula; es decir, si un partido político obtiene un $30 \%$ de la votación en una elección de diputados, a dicho partido le deberán corresponder un $30 \%$ de los diputados del total de la cámara. Es cierto que nuestro sistema, al ser mixto (mayoría relativa y representación proporcional), con participación de muchas opciones en la contienda, puede llevar a extremos de la sobre representación o sub representación, para lo cual la legislación tiene disposiciones o reglas que prohíben que los casos de la sobre y sub representación no se extralimiten de ocho puntos porcentuales.

\section{Requisitos para contender en la elección por el principio de representación proporcional}

La elección de las curules que se disputan bajo el principio de la representación proporcional requieren, siempre, del cumplimiento de una serie de requisitos que podemos encontrar en el texto constitucional del artículo 54 y que después es reflejado en las legislaciones secundarias federal y estatales.

Xihmai 58 
José Arturo Sosa Echeverría

El nuevo mecanismo para la integración de los congresos con diputados de representación proporcional, una fórmula más democrática

Revista Xihmai XIII (26), 51-78, julio-diciembre 2018

De manera general podemos advertir que la posibilidad de contender en esta elección requiere de tres requisitos esenciales:

1. Un registro mínimo de fórmulas en la elección por el principio de mayoría relativa que para el caso de la elección de diputados al Congreso de la Unión deberá alcanzar cuando menos doscientos registros.

2. Obtener un porcentaje mínimo de la votación, que en el caso en mención es de por lo menos el $3 \%$ del total de la votación válida emitida, que corresponde a la que resulte de deducir de la suma de todos los votos depositados en las urnas los votos nulos y los correspondientes a los candidatos no registrados (Ley General de Instituciones y Procedimientos Electorales, art. 15).

3. Registrar una lista de fórmulas de candidatos por ese principio que en el caso de la citada elección federal, se divide en cinco listas regionales de cuarenta fórmulas cada una, una por cada circunscripción electoral plurinominal.

Adicionalmente, y una vez cumplidos los anteriores requisitos, la obtención de diputaciones plurinominales podrá restringirse o incluso negarse en las siguientes hipótesis:

1. Cuando las diputaciones obtenidas por ambos principios exceda de un número tal que represente una mayoría calificada ${ }^{1}$ del órgano legislativo al que se incorporarán.

2. Cuando el número de diputados obtenidos por ambos principios representen un porcentaje del total de la Cámara que exceda en ocho puntos a su porcentaje de votación.

Otro elemento más a considerar en este capítulo se refiere a la prohibición relativa en el sentido de que ningún partido político debe contar con un número tal de diputaciones por ambos principios que pudiera otorgarle una mayoría calificada, por lo que, en el orden federal, se establece que ningún partido político podrá contar con más de trescientos diputados por ambos principios, lo que representa el $60 \%$ de los diputados, situación que reproducen las legislaturas locales con base en el número de diputados que para cada caso establezca la Constitución Política estatal.

\footnotetext{
${ }^{1}$ Según el Sistema de información legislativa, el concepto de mayoría calificada es aquélla donde se exige un porcentaje especial de votación... En el Congreso mexicano corresponde a las dos terceras partes, cuando menos, de los legisladores que se encuentran presentes en el salón de plenos de alguna de las cámaras al momento de tomar una decisión o realizar una votación. Considerando que la Cámara de Diputados está integrada por quinientos legisladores, se requieren 334 votos para alcanzar una mayoría calificada -o un número menor, según el total de asistentes a la sesión-; en la Cámara de Senadores se requerirían 85 de 128 legisladores para lograr dicha mayoría.
}

Xihmai 59 
José Arturo Sosa Echeverría

El nuevo mecanismo para la integración de los congresos con diputados de representación proporcional, una fórmula más democrática

Revista Xihmai XIII (26), 51-78, julio-diciembre 2018

\section{Asignación de curules bajo el principio de la representación proporcional}

Una vez reunidos los requisitos mencionados, el órgano electoral correspondiente procederá a hacer la asignación de las diputaciones que correspondan a cada instituto político con base en sus porcentajes de votación.

Para ello, la Ley General de Instituciones y Procedimientos Electorales desarrolla una fórmula de proporcionalidad pura, integrada por los siguientes elementos: 1 . Cocientes $^{2}$ de distribución; 2. Resto mayor. Fórmula que de manera general reproducen las legislaciones locales de la materia.

Atendiendo al concepto trasunto, el cociente representa el resultado de dividir un número entre otro, lo que nos arroja el resultado de saber cuántas veces cabe un número menor en otro mayor.

Este elemental sistema es el utilizado, en primer lugar, para la aplicación de las fórmulas de asignación de las diputaciones por el principio de representación proporcional. El número mayor se obtiene de la suma de los votos obtenidos por los partidos políticos y el número menor se obtiene del número de diputaciones plurinominales a repartir entre los contendientes.

Aplicada esa operación aritmética de dividir el número total de votos obtenidos por los partidos entre las diputaciones por repartir, al resultado obtenido la ley le denomina como cociente de distribución que, dicho de una manera muy simple, equivale a lo que cuesta cada diputado de representación proporcional a los partidos políticos, solo que ese costo es en votos; es decir, tantas veces tenga un partido político el cociente de distribución en votos, ese mismo número de diputados deberá obtener por el principio de representación proporcional (cuidando siempre de no caer en el caso de la sobre representación mencionada).

La siguiente manera desarrollada por la ley para concluir con la asignación denominada resto mayor, se presenta en el momento en que, aplicada la fórmula de cocientes, resten diputados por repartir; entonces, después de haber dividido el número de votos de cada partido político entre el cociente de distribución y obtenido el resultado de dicha operación aritmética, quedarán siempre cifras adicionales a los números enteros; dichas cifras son las que se utilizarán para

${ }^{2} \mathrm{El}$ concepto de cociente, término cuyo origen se remonta al vocablo latino quotiens (de quot, "cuantos"), tiene dos grandes aplicaciones. En el campo de la matemática, se conoce como cociente al resultado al que se llega tras dividir un número por otro. En este sentido, el cociente sirve para indicar qué cantidad de veces el divisor está contenido en el dividendo (Pérez Porto y Gardey, 2012).

Xihmai 60 
José Arturo Sosa Echeverría

El nuevo mecanismo para la integración de los congresos con diputados

de representación proporcional, una fórmula más democrática

Revista Xihmai XIII (26), 51-78, julio-diciembre 2018

otorgar el resto de los diputados por repartir hasta alcanzar el número total equivalente a la representación proporcional de que se trate.

\section{Forma tradicional de obtención de curules por el principio de representación proporcional}

A partir de la instauración en el marco legal mexicano de la figura de la representación proporcional, la asignación de las curules que obtienen los partidos políticos por dicho principio se ha venido dando de la misma forma.

Como ya se mencionó, la legislación electoral del país impone una serie de requisitos a satisfacer para contender en la elección de las diputaciones que acceden al cargo bajo el principio de la representación proporcional; el correspondiente al registro de la lista única de fórmulas de candidatos a obtener dichos espacios provenía, eminentemente, de las cúpulas partidarias; esto es, en ejercicio de la libre configuración intrapartidaria, los dirigentes de los institutos políticos ordenaban y presentaban la lista de los candidatos para su registro ante el órgano electoral respectivo, según las conveniencias propias del partido o los merecimientos de quienes eran incluidos en dicha lista, incluso, en algunos casos, provenían de procesos electivos internos de los propios partidos políticos.

En ese contexto, sea cual fuere la forma de acceder a la lista de fórmulas de candidatos a ocupar las curules que por el principio de representación proporcional correspondieran a cada partido político, podíamos apreciar que en todas las disposiciones electorales del país solamente estaba prevista la configuración y presentación para su registro de la lista única de aspirantes a tales diputaciones; se dejaba, entonces, a los institutos políticos registrados ante los organismos electorales la libertad de decidir la manera de seleccionar a sus candidatos, sin importar si el electorado pudiera tener o no simpatía o antipatía por sus propuestas, ya que el voto que les daba la posibilidad de ocupar las diputaciones plurinominales no lo asignaba el ciudadano de manera directa al candidato de esa lista, pues el voto directo se dirigía a la opción que el partido había seleccionado para contender en la elección por el principio de mayoría relativa, por lo tanto, el acceso de estas figuras representaba una decisión eminentemente intrapartidaria sin contar necesariamente con la anuencia del electorado.

Es preciso mencionar que en la asignación de las diputaciones, se sigue el orden que tuviesen los candidatos en las listas correspondientes, por lo que los registrados en las primeras posiciones de las listas tendrán mayores posibilidades de acceder a la Cámara de Diputados. 
José Arturo Sosa Echeverría

El nuevo mecanismo para la integración de los congresos con diputados

de representación proporcional, una fórmula más democrática

Revista Xihmai XIII (26), 51-78, julio-diciembre 2018

\section{La nueva forma de acceso a las diputaciones de representación proporcional, Listas A y B}

Como ha quedado de manifiesto, para la integración de la representación proporcional en los órganos legislativos del país, tradicionalmente se optaba por el registro de una lista única presentada por los partidos políticos.

En los mencionados términos tradicionales, pasada la jornada electoral en la que los ciudadanos emitieron su voto para decidir de manera directa a una parte de los integrantes del órgano legislativo correspondiente (diputados electos por el principio de mayoría relativa), los institutos o consejos electorales respectivos determinaban cuántas diputaciones de representación proporcional correspondían a cada partido político que hubiese cubierto los requisitos legales atinentes, por lo que, una vez resuelto numéricamente las diputaciones a otorgar, la asignación se daba de conformidad al orden descendente mencionado en la lista registrada, comenzando por quien ocupaba el primer lugar y así sucesivamente hasta alcanzar el número de curules que les correspondiera.

Dicho mecanismo de asignación ha venido cambiando en los últimos años, ya que diferentes legislaciones en el país (todas ellas estatales, ya que la Ley General de Instituciones y Procedimientos Electorales no lo prevé así) han adoptado un componente distinto al tradicional.

Las entidades federativas que han decidido migrar del sistema cotidiano y único hasta la actualidad establecen, en lo general, un sistema de doble lista.

La primera lista o lista "A". El nuevo procedimiento sigue manteniendo la posibilidad de que los partidos políticos contendientes en una elección legislativa inscriban a las fórmulas de candidatos a elegirse bajo el principio de representación proporcional con base en su normatividad interna, es decir, conservan esa parte del sistema tradicional que permite a los institutos políticos la configuración de una lista de candidatos que ellos mismos han venido confeccionando en base a sus intereses o normatividades internas.

La segunda lista o lista "B". El novedoso mecanismo establece la conformación de una segunda lista de fórmulas de candidatos que, junto con la registrada originalmente, se habrán de disputar las curules a asignar.

Cada legislación tiene sus particularidades, a las que me habré de referir más adelante, pero de manera general se advierte, en todas esas disposiciones, la inclusión de un componente democrático, un elemento que privilegia la voluntad ciudadana al emitir su sufragio de manera directa hacia determinada fórmula de

Xihmai 62 
José Arturo Sosa Echeverría

El nuevo mecanismo para la integración de los congresos con diputados de representación proporcional, una fórmula más democrática

Revista Xihmai XIII (26), 51-78, julio-diciembre 2018

candidatos en la conformación del legislativo bajo el principio de la representación proporcional.

La razón es que la segunda lista o lista "B" se va a construir a partir de las fórmulas de candidatos que fueron registrados para contender en la elección bajo el principio de mayoría relativa, candidatos que realizaron actos de campaña y a quienes de manera directa el electorado otorgó su voto, en el entendido de que no fueron favorecidos por la mayoría de los sufragios; esta segunda lista se conforma, pues, con las fórmulas no ganadoras de la elección directa, en un orden descendente de acuerdo con los porcentajes de votación obtenidos y quienes, después de realizadas las operaciones y desarrolladas las fórmulas de asignación, se habrán de incorporar a la repartición de los espacios en el poder legislativo, alternándose con las fórmulas de candidatos inscritos por los partidos políticos.

Las entidades federativas del país que han adoptado el nuevo mecanismo para la conformación de las diputaciones por el principio de representación proporcional son las que a continuación se mencionan y los artículos de sus respectivas legislaciones se transcriben en la parte que establece el doble mecanismo de asignación.

Aguascalientes. El artículo 150 del Código Electoral del Estado de Aguascalientes dispone:

La lista estatal de candidatos a diputados de representación proporcional que los partidos presenten deberá integrarse de la siguiente manera: I. En el primero, cuarto, quinto, séptimo, octavo y noveno lugar de la lista se inscribirán a las fórmulas de candidatos que el partido correspondiente postule, repartiendo tales posiciones alternadamente entre candidatos de distinto género, y II. El segundo, tercero y sexto sitio de la lista se reservará para las fórmulas de candidatos de los partidos políticos que no obtuvieron el triunfo por el principio de mayoría relativa, asignándolos en orden decreciente, a las que hubieren obtenido los más altos porcentajes de votación en su distrito electoral.

Como puede observarse en el caso del estado de Aguascalientes, no se confeccionan dos listas, sino que aparece una sola presentada por el partido político con candidatos previamente definidos en los lugares $1^{\circ}, 4^{\circ}, 5^{\circ}, 7^{\circ}, 8^{\circ} \mathrm{Y}$ $9^{\circ}$, dejando sin presentar fórmula de candidatos para su registro en los espacios $2^{\circ}, 3^{\circ}$ y $6^{\circ}$ para los candidatos que contienden en la elección de mayoría relativa, que no obtienen el triunfo y que se ordenan de mayor a menor con base en su porcentaje de votación.

En las mencionadas condiciones, si a un partido político le corresponde más de una diputación por el principio de representación proporcional, la segunda y 
José Arturo Sosa Echeverría

El nuevo mecanismo para la integración de los congresos con diputados de representación proporcional, una fórmula más democrática

Revista Xihmai XIII (26), 51-78, julio-diciembre 2018

tercera curules provendrán de los candidatos que participaron en la elección de mayoría relativa, candidatos que aunque no lograron el triunfo, los electores les otorgaron votos en cantidades considerables que les permiten ser compensados con un espacio en el Congreso.

Baja California. En el caso de este estado, la Constitución Política local dispone lo siguiente:

ARTÍCULO 15.- La asignación de los Diputados por el principio de representación proporcional que le correspondan a cada partido político, se hará por el Instituto Estatal Electoral de acuerdo con el procedimiento que se establezca en la Ley, y atendiendo lo siguiente: I.- Para que los partidos políticos tengan este derecho deberán: a) Participar con candidatos a Diputados por el principio de mayoría relativa en por lo menos el cincuenta por ciento de los distritos electorales; b) Haber obtenido por lo menos el tres por ciento de la votación válida emitida en la elección de Diputados por el principio de representación proporcional, y c) Haber obtenido el registro de la lista de cuatro candidatos a Diputados por el principio de representación proporcional. Corresponde a cada partido político o coalición, en su caso, determinar si la primera asignación que le corresponda por este principio, será con base en la lista registrada o por porcentaje de votación válida, especificándolo en el periodo de registro de la lista de candidatos o en su caso, en el convenio de coalición, ante el Instituto Estatal Electoral. II.- El Instituto Estatal Electoral una vez verificados los requisitos de la fracción anterior, asignará un Diputado a cada partido político que tenga derecho a ello. Esta primera asignación corresponderá a los candidatos a diputados de las listas previamente registradas ante la autoridad electoral o los que tengan mayor porcentaje de votación válida en el distrito y que no hayan obtenido constancia de mayoría. En caso de que el número de partidos políticos sea mayor que el de diputaciones por asignar, éstas se otorgarán a los que tengan mayor porcentaje en orden descendente hasta agotarse; [...]

Por su parte, el artículo 27 de la Ley Electoral del Estado de Baja California dispone:

El Consejo General hará la asignación de diputados a cada partido político conforme al resultado obtenido en los artículos anteriores, en los siguientes términos: I. Determinará que candidatos a Diputados de cada partido político no obtuvieron la constancia de mayoría; debiendo identificar, en el caso de coalición, a qué partido político pertenece el candidato en coalición. II. Elaborará una lista en orden descendente de cada partido político con los candidatos que no hayan obtenido la constancia de mayoría, de acuerdo a su porcentaje de votación válida en el distrito respectivo. El porcentaje se tomará hasta diezmilésimas, sin redondear la última cifra. Se entenderá por votación valida, la sumatoria de los votos obtenidos por los partidos políticos en el ámbito territorial de que se trate; III. Si dos o más candidatos de un partido político tienen el mismo porcentaje en la lista, hasta antes del séptimo lugar, el Consejo General le solicitará al partido político, para que dentro de las veinticuatro horas siguientes a que sea notificado, determine el lugar que le corresponderá; IV. Si dentro del plazo señalado en la fracción anterior el partido político no da respuesta, el

Xihmai 64 
José Arturo Sosa Echeverría

El nuevo mecanismo para la integración de los congresos con diputados de representación proporcional, una fórmula más democrática

Revista Xihmai XIII (26), 51-78, julio-diciembre 2018

Consejo General procederá a determinarlo mediante sorteo; V. Una vez determinada la lista anterior se procederá a su intercalación con la que hubiere registrado cada partido político por el principio de representación a que se refiere el inciso c) de la fracción I del artículo 15 de la Constitución del Estado; atendiendo lo siguiente: a) La intercalación iniciará con el que ocupe el primer lugar de acuerdo a la lista registrada por el principio de representación proporcional, si así lo hubiera determinado el partido político en los plazos y condiciones previstos en esta ley, seguido de quien ocupe el primer lugar en la lista obtenida de la fracción II de este artículo, continuando de manera alternada en el orden de prelación resultante, en cada caso, para lograr una lista de hasta ocho diputaciones a asignar. b) Si el partido político no hubiere realizado la determinación a que se refiere el inciso anterior, la intercalación iniciará con el que ocupe el primer lugar en la lista obtenida de la fracción II de este artículo, seguido de quien ocupe el primer lugar de acuerdo a la lista registrada por el principio de representación proporcional, continuando de manera alternada en el orden de prelación resultante, en cada caso, para lograr una lista de hasta ocho diputaciones a asignar. c) En los casos, de partidos políticos que hubieren participado coaligados, y sus candidatos que conformen la lista en base al porcentaje de votación valida no fueran suficientes para la intercalación de hasta ocho diputaciones, la intercalación se hará hasta donde fuera posible continuado con los de la lista de representación proporcional registrada. d) En los casos, de partidos políticos que hubieren participado coaligados, y no cuenten con candidatos para integrar la lista en base al porcentaje de votación valida, la asignación que les corresponda se hará de la lista de representación proporcional registrada en términos de ley. En caso de que un partido político obtenga trece o más diputaciones por el principio de mayoría relativa, la lista se integrará sólo con aquellos candidatos que no obtengan constancia de mayoría, junto con los registrados en la lista de representación proporcional, respetando en todo momento lo señalado en los incisos anteriores, con excepción de los lugares que no podrán ser ocupados por aquéllos, recorriendo en dichos espacios a éstos, y VI. En caso de que la asignación recaiga en quien este inhabilitado o no reúna los requisitos para ser electo la asignación deberá ser cubierta por el suplente de la fórmula respectiva. Si éste último también resulta inhabilitado o no reúne los requisitos para ser electo, se asignará aquella fórmula de candidatos del mismo partido político que siga en el orden de la lista.

Primera lista. Como puede advertirse en el caso de Baja California, los partidos políticos tienen la obligación de presentar para su registro una lista de cuatro fórmulas de candidatos a elegirse bajo el principio de representación proporcional $\mathrm{y}$, en ese mismo acto, tienen la posibilidad de definir si la lista registrada en ese momento sería, en su caso, la primera opción para empezar con las asignaciones que correspondieran al partido político, o bien, por la lista que se conformaría con base en los resultados obtenidos por los candidatos de mayoría relativa que no hayan logrado el triunfo.

Segunda lista. Pasada la jornada electoral, el Consejo General del organismo público local electoral, llegado el momento de la asignación, procede a conformar una lista por cada instituto político, en la que ordena descendentemente, por

Xihmai 65 
José Arturo Sosa Echeverría

El nuevo mecanismo para la integración de los congresos con diputados de representación proporcional, una fórmula más democrática

Revista Xihmai XIII (26), 51-78, julio-diciembre 2018

porcentaje de votación obtenido, las fórmulas de candidatos que no obtuvieron el triunfo en la elección de mayoría relativa.

Posteriormente, el Consejo General intercala ambas listas, comenzando por la que al momento del registro de la primera lista haya definido el partido político o bien, de no haber definido cuál sería la primer lista a considerar para la asignación correspondiente, deberá de iniciarse con la lista que el Consejo General haya ordenado con los candidatos registrados para contender en la elección de mayoría relativa y que no hayan obtenido el triunfo en dicha elección.

Como puede apreciarse en el caso de Baja California, el partido político tiene la posibilidad de elegir por cuál de las dos listas se iniciaría la asignación de las curules que les correspondan por el principio de representación proporcional; no obstante, si el partido, al momento del registro de las candidaturas, fue omiso en la designación de la lista por la que deba comenzar la asignación, el Consejo General deberá iniciar por la lista que incluye a los candidatos que contendieron en la elección de mayoría relativa y que no hayan obtenido el triunfo en aquella.

Chihuahua. La Ley Electoral de ese estado establece lo siguiente:

Artículo 17. 1) Para la asignación de diputados electos por el principio de representación proporcional, cada partido político deberá registrar una lista de seis fórmulas de candidatos propietarios y suplentes, la cual no podrá contener más del $50 \%$ de candidatos de un mismo género. Cada fórmula deberá ser del mismo género. El incumplimiento de este precepto dará lugar a la negativa del registro de la referida lista, la que, en su caso, podrá subsanarse dentro del lapso de registro señalado para ese efecto. 2) Para garantizar la pluralidad representativa en el Congreso del Estado, se asignará en una primera ronda una diputación a cada partido político que haya obtenido por lo menos el $3 \%$ de la votación estatal válida emitida. Si aún quedaren diputaciones por asignar, en una segunda ronda se otorgará otra diputación a cada partido político que haya obtenido más del $5 \%$ de la votación. Si aún quedaren diputaciones por asignar, en una tercera ronda se otorgará otra diputación a cada partido político que haya obtenido más del $10 \%$ de la votación. Si aún quedaren diputaciones por asignar, en una cuarta ronda se asignará otra diputación a cada partido político que haya obtenido más del $20 \%$ de la votación. Si agotado este procedimiento, aún quedaren diputaciones por asignar, éstas se otorgarán por rondas de asignación, de una en una y en orden decreciente del porcentaje de votación obtenido por los partidos políticos hasta agotar su totalidad. 3) Las diputaciones de representación proporcional que correspondan a cada partido político se asignarán alternada y sucesivamente: en primer lugar, utilizando el sistema de listas previamente registradas por aquellos conforme a esta Ley, y, en segundo lugar, atendiendo a los más altos porcentajes obtenidos en su distrito por cada uno de los candidatos del mismo partido político, de la votación estatal válida emitida.

Xihmai 66 
José Arturo Sosa Echeverría

El nuevo mecanismo para la integración de los congresos con diputados de representación proporcional, una fórmula más democrática

Revista Xihmai XIII (26), 51-78, julio-diciembre 2018

Primera lista. Chihuahua establece también el registro de la lista tradicional conformada por seis fórmulas de candidatos registrada por cada partido político, la que al momento de la asignación sería utilizada en primer término.

Segunda lista. Determina también la ley local, la conformación de una segunda lista, la de los candidatos provenientes de la elección de mayoría relativa que sin haber obtenido el triunfo hayan alcanzado los mejores porcentajes de la votación en su distrito; esta lista se ocuparía en segundo lugar con relación a la primera lista.

Ciudad de México. El Código de Instituciones y Procedimientos Electorales de la Ciudad de México dispone:

Artículo 24. Para la asignación de Diputadas y Diputados electos por el principio de representación proporcional se tendrán en cuenta los conceptos y principios siguientes: ...III. Lista "A": Relación de diecisiete fórmulas de candidaturas a las diputaciones: propietario y suplente del mismo género, listados en orden de prelación alternando fórmulas de género distinto de manera sucesiva, a elegir por el principio de representación proporcional, de las cuales 4 deberán estar integradas por jóvenes de 18 a 35 años; IV. Lista "B": Relación de diecisiete fórmulas de candidaturas a las diputaciones que no lograron el triunfo en la elección por el principio de mayoría relativa del distrito en que participaron, pero que alcanzaron a nivel distrital los mayores porcentajes de la votación local emitida, comparados respecto de otras fórmulas de su propio partido en esa misma elección; con la finalidad de garantizar la paridad de género, una vez que se determinó el primer lugar de ésta lista, el segundo lugar será ocupado por la fórmula del otro género con mayor porcentaje de la votación local emitida, e irán intercalando de esta manera hasta concluir la integración de la lista. V. Lista Definitiva, es el resultado de intercalar las fórmulas de candidatos de las Listas "A" y "B", que será encabezada siempre por la primera fórmula de la Lista "A". Tal intercalado podrá generar bloques de hasta dos fórmulas del mismo género, pero de diferente lista de origen.

Artículo 27. Para la asignación de curules por el principio de representación proporcional se observarán las siguientes reglas:... V. Para la asignación de diputaciones de representación proporcional del Congreso de la Ciudad de México se utilizará la fórmula atendiendo las reglas siguientes: a) Se intercalarán las fórmulas de candidatos y candidatas de ambas listas, iniciándose con los candidatos de la Lista "A".

Lista "A". Para el caso de la Ciudad de México, la legislación electoral local establece la conformación de una primera lista denominada lista "A", que se compone de diecisiete fórmulas de candidatos listados en orden numérico y que corresponde a la que cada instituto político participante registra en la contienda electoral distrital; esta lista es la primera que debe emplear el organismo público local electoral para la asignación de las diputaciones por el principio de representación proporcional. 
José Arturo Sosa Echeverría

El nuevo mecanismo para la integración de los congresos con diputados de representación proporcional, una fórmula más democrática

Revista Xihmai XIII (26), 51-78, julio-diciembre 2018

Lista "B". Se compone con las diecisiete fórmulas de candidatos que no alcanzaron el triunfo en la elección de mayoría relativa y que obtuvieron a nivel distrital los mayores porcentajes de votación, comparados con otras fórmulas de su propio partido; la opción " $\mathrm{B}$ " es la ocupada en segundo término para la asignación de las curules plurinominales.

Guanajuato. La Constitución Política de esta entidad establece la conformación de listas diferenciadas para la integración de la legislatura local con los candidatos electos bajo el principio de la representación proporcional, lo que se advierte de la lectura del siguiente precepto constitucional:

Artículo 44.- La elección de los catorce diputados según el principio de representación proporcional mediante el sistema de listas, se regulará a lo que en lo particular disponga la Ley y se sujetará a las bases generales siguientes: I.- Para obtener el registro de sus listas de candidatos, el partido político que lo solicite deberá acreditar que participa con candidatos por mayoría relativa, en por lo menos quince de los distritos uninominales y que cuenta con registro como partido político nacional o estatal. La lista de candidatos de cada partido político se integrará con: a) Las propuestas que los partidos políticos presenten; y b) los candidatos de las fórmulas por el principio de mayoría relativa que no hayan obtenido constancia de mayoría pero sean los que hayan obtenido el mayor porcentaje de votación del partido político que los postuló. La asignación de los diputados que correspondan a cada partido político la hará el organismo público electoral local de manera alternada cada tres asignaciones de entre las opciones que integran la lista anterior, iniciando por las propuestas contenidas en el inciso a); en la forma y términos que señale la Ley de la materia.

La Ley de Instituciones y Procedimientos Electorales para el estado de Guanajuato dispone:

Artículo 273. La asignación de diputados por el principio de representación proporcional que correspondan a cada partido político la hará el Consejo General atendiendo además a lo siguiente: I. Determinado el número de diputados que le corresponda a cada partido político, se procederá a ordenar, en forma descendente respecto al porcentaje obtenido en el distrito, las fórmulas de candidatos a diputados uninominales de un mismo instituto político que no hayan obtenido constancia de mayoría; II. Se integrará la lista de candidatos a diputados por el principio de representación proporcional de cada partido político en dos apartados conteniendo: a) Las ocho fórmulas conformadas de propietario y suplente designadas en orden de prelación por cada partido político, y b) Las fórmulas de candidatos a diputados por el principio de mayoría relativa resultantes de la operación señalada en la fracción anterior. III. Las diputaciones se adjudicarán, de entre las fórmulas que integran la lista señalada en la fracción anterior, de manera alternada cada tres asignaciones, iniciando con las tres primeras fórmulas contenidas en el inciso a), prosiguiendo con las tres

\section{Xihmai 68}


José Arturo Sosa Echeverría

El nuevo mecanismo para la integración de los congresos con diputados de representación proporcional, una fórmula más democrática

Revista Xihmai XIII (26), 51-78, julio-diciembre 2018

primeras fórmulas a que se refiere el inciso b), continuando hasta completar el número de diputados que le corresponda a cada partido político.

Primera lista. Se conforma, en términos de lo establecido en la Constitución Política local, con las propuestas que los partidos políticos presenten para su registro ante el organismo electoral de la entidad.

Segunda lista. Es la integrada por las fórmulas de candidatos por el principio de mayoría relativa que no obtengan constancia de mayoría pero que hayan obtenido el mayor porcentaje de votación del partido político que los postuló.

La asignación que realice la autoridad electoral local, una vez determinado el número de curules que bajo el principio de representación proporcional corresponda a cada instituto político participante en la contienda, la hará de forma alternada, sólo que en el caso de este estado de la República, toma tres fórmulas de la primera lista y si hubiera más diputaciones por asignar, las dispone de la segunda lista, lo que hace inequitativo, en mi concepto, el procedimiento de asignación al establecer los dos mecanismos para arribar a las curules plurinominales.

Hidalgo. El Código Electoral del Estado de Hidalgo establece la conformación de su legislatura a nivel de los diputados electos por el principio de representación proporcional a través de dos listas diferenciadas: la primera es denominada lista "A" y está integrada por los candidatos propuestos por el partido para ese efecto; la segunda, llamada lista "B", se integrará con los candidatos que hayan participado en la elección por el principio de mayoría relativa y que no hayan obtenido el triunfo; así lo narran los artículos siguientes:

Artículo 207. Una vez resueltos por las instancias jurisdiccionales competentes los medios de impugnación presentados respecto de los cómputos distritales, declaración de validez de las elecciones y entrega de las constancias de mayoría, el Consejo General del Instituto Estatal Electoral procederá a asignar doce Diputados por el principio de representación proporcional, en la asignación de los Diputados electos por el principio de representación proporcional tendrán derecho a participar los Partidos Políticos debidamente registrados, que cumplan los requisitos siguientes: I. Registrar una lista "A", con 12 fórmulas de candidatos a Diputados a elegir por el principio de representación proporcional, conforme a lo que se estipula en el presente Código;... Artículo 208. Para la asignación de Diputados electos por el principio de representación proporcional se tendrán en cuenta los conceptos y principios siguientes: I. Lista "A": Relación de doce fórmulas de candidatos a Diputados propietario y suplente del mismo género, listados en orden de prelación alternando fórmulas género de manera sucesiva, a elegir por el principio de representación proporcional; II. Lista "B": Relación de las fórmulas de candidatos a Diputados que no lograron el triunfo en la elección por el principio de mayoría relativa del distrito en que participaron, pero 
José Arturo Sosa Echeverría

El nuevo mecanismo para la integración de los congresos con diputados de representación proporcional, una fórmula más democrática

Revista Xihmai XIII (26), 51-78, julio-diciembre 2018

que alcanzaron a nivel distrital los mayores porcentajes de la votación válida emitida, comparados respecto de otras fórmulas de su propio partido en esa misma elección; con la finalidad de garantizar la paridad de género, una vez que se determinó el primer lugar de ésta lista, el segundo lugar será ocupado por la fórmula del otro género con mayor porcentaje de la votación efectiva, e irán intercalando de esta manera hasta concluir la integración de la lista. Al principio de la lista B se deberá ubicar a una persona del sexo diverso al del primer lugar de la lista A".

Lista "A". Hidalgo es, entonces, otra de las entidades federativas del país que ha optado por la aplicación de un nuevo método para la integración de su Congreso en cuanto a la representación proporcional se refiere; como ha podido apreciarse, la lista "A" proviene de la decisión partidista a través de la designación de doce fórmulas presentadas de manera descendente y alternadas por géneros.

Lista "B". Asimismo la legislación electoral local de este estado instaura una segunda lista de candidatos, la lista "B", que se conforma con los candidatos en la elección de mayoría relativa que no lograron el triunfo y que se ordenan con base en sus porcentajes de votación.

La asignación la hará el Consejo General del Instituto Estatal Electoral iniciando por el primer integrante de la lista " $A$ " para seguir en segundo orden por el primero de la lista "B" (debiendo ser de género distinto), y así alternadamente hasta alcanzar el número de curules que corresponda a cada uno de los partidos políticos.

Estado de México. El Código Electoral del Estado de México dispone:

Artículo 369. La asignación de diputados de representación proporcional que corresponda a cada partido político conforme al artículo anterior, se hará alternando, los candidatos que aparezcan en la lista presentada por los partidos políticos y los candidatos que no habiendo obtenido la mayoría relativa, hayan alcanzado la votación, en números absolutos, más alta de su partido por distrito. Esto en el orden en que se presenten ambos... En todo caso, la asignación se iniciará con la lista registrada en términos de este Código.

De lo establecido en la porción normativa trasunta concluimos que una vez realizadas las operaciones aritméticas procedentes para determinar el número de curules que correspondan a cada partido político, el organismo público local electoral asignará las diputaciones de representación proporcional a los partidos políticos con base en dos listas:

Primera lista. Corresponde a la que registren los partidos políticos para el efecto de contender mediante ese principio.

Xihmai 70 
José Arturo Sosa Echeverría

El nuevo mecanismo para la integración de los congresos con diputados de representación proporcional, una fórmula más democrática

Revista Xihmai XIII (26), 51-78, julio-diciembre 2018

Segunda lista. Se conforma con los candidatos que no lograron la mayoría relativa ordenados de mayor a menor con base en los números obtenidos en la elección correspondiente.

La asignación la hará el organismo público local electoral iniciando con la primera lista y alternándola con la segunda lista hasta llegar al total de curules por repartir.

Nayarit. La Ley Electoral del Estado de Nayarit establece:

Artículo 21.- Para la elección de los Diputados según el principio de Representación Proporcional, se constituirá una sola circunscripción electoral en el Estado. I. Para concurrir a la asignación de Diputados por este principio, los partidos políticos y coaliciones deberán acreditar: a) Los partidos políticos, que participan con fórmulas de candidatos a Diputados por el sistema de Mayoría Relativa en por lo menos las dos terceras partes de los distritos electorales uninominales; las coaliciones, cuando acrediten el cumplimiento de lo establecido por los artículos 64 segundo párrafo, y 69 del presente ordenamiento; b) Haber registrado listas estatales de candidatos para esta elección de hasta un número de doce ciudadanos por cada partido político o coalición. Opcionalmente, los partidos políticos o coaliciones podrán presentar esta lista con un número de seis ciudadanos que ocuparán los números nones de la lista total de conformidad con el orden que presenten los partidos políticos y coaliciones, en los términos de la fracción $\mathrm{V}$ del artículo 22 de la presente ley y los seis restantes, corresponderán a los números pares de la lista, mismos que serán cubiertos por el organismo electoral, una vez realizada la elección, con los candidatos de mayoría relativa registrados por el partido político respectivo, que no hayan alcanzado el triunfo de mayoría relativa y los cuales serán ordenados en forma decreciente conforme al porcentaje de votos obtenidos en relación directa al total de votos computados en la elección distrital respectiva. Los partidos políticos o coaliciones tendrán la potestad de presentar la lista de manera invertida a lo previsto en el párrafo anterior. Una vez registrada la lista de candidatos por alguna de las anteriores opciones, el partido político o coalición postulante no podrá optar por otra. Artículo 22.- A los partidos políticos o coaliciones que obtengan cuando menos el 1.5 por ciento de la votación total estatal en la elección de Diputados de Mayoría Relativa, les será asignado un Diputado por el principio de Representación Proporcional, con excepción de aquel al que se le hubiere otorgado las constancias de mayoría y validez de la totalidad de los distritos electorales. Si aún existieren diputaciones por asignar, se procederá de la siguiente manera:... V. En todos los casos, las asignaciones se harán en el orden que determinen los partidos políticos o coaliciones, de su lista estatal registrada, una vez concluido en los términos de esta ley, el cómputo y hecha la declaración de validez para esta elección.

El estado de Nayarit intenta incluir en su legislación un modelo democrático en la conformación de la legislatura local con respecto a las fórmulas de candidatos electos bajo el principio de representación proporcional al establecer la posibilidad de que, de la lista que presenten para su registro, se dejen los números pares a los candidatos que contiendan en la elección bajo el principio de mayoría Xihmai 71 
José Arturo Sosa Echeverría

El nuevo mecanismo para la integración de los congresos con diputados de representación proporcional, una fórmula más democrática

Revista Xihmai XIII (26), 51-78, julio-diciembre 2018

relativa y que no hayan obtenido el triunfo; sin embargo, deja a los partidos políticos la posibilidad de tomar o no esa decisión; de no hacerlo, siguen con la misma posibilidad de completar su lista con candidatos provenientes del propio instituto político sin dar posibilidades a los candidatos que participaron en la verdadera contienda electoral, por lo que excluye a los candidatos que a través de sus campañas electorales llevaron votos a su partido político.

Oaxaca. El Código de Instituciones Políticas y Procedimientos Electorales para el Estado de Oaxaca dispone:

Artículo 153. 4. El registro de los candidatos a diputados locales por el principio de representación proporcional, se realizará mediante cualquiera de las siguientes opciones: I.- Por listas de diecisiete candidatos a diputados propietarios y suplentes por el principio de representación proporcional; y II.- Por relaciones de hasta veinticinco candidatos a diputados por el principio de representación proporcional, conformadas con los mismos candidatos de mayoría relativa. 5. Al momento del registro de las listas de candidatos a diputados por el principio de representación proporcional, los partidos políticos precisarán por cuál de las dos opciones registran dichas listas. En caso de no precisar cualquiera de las dos opciones, se entenderá que eligió la opción contenida en la fracción primera del párrafo cuarto de este artículo. Artículo 251 El Consejo General hará el cómputo de votaciones de la circunscripción plurinominal, para tal efecto se observará lo siguiente:... VIII.- Las diputaciones obtenidas por cada uno de los partidos políticos se asignarán en la forma siguiente: a).- Según el orden en que aparezcan en sus respectivas listas registradas ante el Instituto, si optaron por el procedimiento establecido en el artículo 153 numeral 4, fracción I de este Código; y b).- Según el orden decreciente de la votación obtenida por sus candidatos en la elección por el principio de mayoría relativa, si optaron por el procedimiento establecido en el artículo 153 numeral 4, fracción II de este Código.

La legislación electoral de esta entidad federativa contiene un aspecto relevante: da a los partidos políticos la posibilidad de elegir una de dos opciones en cuanto a su registro de fórmulas de candidatos por el principio de representación proporcional. Pueden optar por la forma tradicional de una lista proveniente del partido político con independencia de la que hayan registrado en la contienda bajo el principio de mayoría relativa, o bien, elegir la opción de obtener su relación de fórmulas con las registradas para la elección por el principio de mayoría relativa.

Lo singular del caso Oaxaca es que el organismo público electoral local no designa alternadamente entre las dos opciones o las dos listas; en este caso, el partido político decide, desde el momento del registro de sus candidaturas a diputados locales por el principio de representación proporcional, cuál de las dos opciones deciden para que, llegado el momento, les hagan las asignaciones correspondientes y, de presentarse el caso que el partido político haya sido omiso en cuanto a definir cuál opción deciden, el código comicial de la entidad salva la

Xihmai 72 
José Arturo Sosa Echeverría

El nuevo mecanismo para la integración de los congresos con diputados

de representación proporcional, una fórmula más democrática

Revista Xihmai XIII (26), 51-78, julio-diciembre 2018

omisión definiendo que la asignación se realice conforme a la lista proveniente del partido político.

Yucatán. La Ley de Instituciones y Procedimientos Electorales del Estado de Yucatán dispone:

Artículo 214. Las disposiciones del presente capítulo regulan el procedimiento de registro de candidatos a cargos de elección popular. I. El registro de candidatos a cargos de elección popular se realizará conforme a lo siguiente:... b) Las candidaturas a diputados a elegirse por el principio de representación proporcional se registrarán por medio de listas de 5 candidatos propietarios, alternando los géneros de los candidatos para garantizar el principio de paridad hasta agotar la lista;... Artículo 330. Previamente a la asignación de los diputados electos por el sistema de representación proporcional, el Consejo General del Instituto procederá a integrar una lista de 10 candidatos en orden de prelación, por cada uno de los partidos políticos y coaliciones, que hubieran alcanzado el Porcentaje Mínimo de Asignación, aplicado el siguiente procedimiento: I. Se tendrá por lista preliminar la integrada por los 5 candidatos de representación proporcional a que se refiere el inciso b), fracción I, del artículo 214, de esta Ley, que hubiera registrado el partido político o coalición; II. Se elaborará una segunda lista con los 5 candidatos de mayoría relativa que encabezaron su fórmula, del mismo partido político o coalición, ordenados de manera decreciente de acuerdo a los mayores porcentajes de votación válida que hubieran alcanzado en sus respectivos distritos, con relación a los candidatos de su propio partido político o coalición que no hubieran ganado la elección;... III. La lista definitiva de los candidatos para la asignación, a que se refiere el primer párrafo de este artículo, se integrará alternando uno a uno, a los relacionados en las listas anteriores, iniciando con el primero de la lista a que se refiere la fracción I de este artículo.

Primera lista. Para el caso del estado de Yucatán, se evidencia que los partidos políticos deben registrar una lista de cinco fórmulas de candidaturas a diputados por el principio de representación proporcional.

Segunda lista. Se forma con los cinco candidatos de mayoría relativa que no lograron el triunfo y que obtuvieron los mayores porcentajes de votación en sus respectivos distritos.

El Consejo General del Instituto Electoral del Estado procede a integrar una lista final de diez candidatos, conformada con la lista preliminar integrada por las cinco fórmulas registradas por los partidos políticos y con la segunda lista formada con los cinco candidatos de mayoría relativa que no lograron el triunfo y que obtuvieron los mayores porcentajes de votación en sus respectivos distritos. Para la conformación de la lista definitiva de diez fórmulas de candidatos a la representación proporcional, se van ir intercalando, uno a uno, los candidatos de ambas listas, comenzando por quien encabece la lista preliminar y continuando por quien encabece la segunda lista, alternadamente hasta su conformación total. 
José Arturo Sosa Echeverría

El nuevo mecanismo para la integración de los congresos con diputados de representación proporcional, una fórmula más democrática

Revista Xihmai XIII (26), 51-78, julio-diciembre 2018

El Consejo General otorgará el número de diputaciones que corresponda a cada partido político y las asignará conforme al orden que hayan quedado en la lista definitiva.

\section{Conclusiones}

Con la inclusión en el sistema político mexicano en 1963 de los Diputados de Partido, comenzó incipientemente una nueva configuración legislativa en nuestro país. Lo que inicia como una graciosa concesión a las minorías, se fue transformando hasta llegar a una configuración legislativa en donde hoy en día no hay una mayoría absoluta en un solo partido político.

La incorporación de los diputados de representación proporcional en 1977 y la paradigmática reforma constitucional del año de 1996, en donde cambian disposiciones e instituciones para hacer los procesos electivos más democráticos, produjeron una nueva percepción sobre la asunción a los cargos públicos en nuestro país, específicamente en la integración de los órganos legislativos.

La posibilidad de que los partidos políticos que no obtenían los triunfos en las elecciones de mayoría relativa, pero que podían integrar los congresos con un número de representantes que se les otorgaba en base a sus porcentajes de votación, promovió la participación política y mejores contiendas electorales.

Los partidos políticos, sabedores de que, aun y cuando no obtuvieran la mayoría de los votos que les representara el acceso directo a los espacios en disputa, acudían a la contienda con la intención de estar mayormente representados y tener la posibilidad de gestar, desde los órganos legislativos, los cambios sustanciales que abonaran con un esquema más democrático en el país.

Es así como la democracia nacional ha avanzado, al grado de que los espacios de representación proporcional que originalmente se atribuían a las decisiones internas (o de cúpula) de los partidos políticos (sin dejar de pasar por las decisiones intrapartidistas), empiezan a tomar un esquema mayormente democrático en la integración de las diputaciones plurinominales.

Originalmente, desde la confección del sistema electoral mixto en nuestro país, la conformación de la representación proporcional provenía de una lista única, presentada y ordenada numéricamente por los partidos políticos, por lo que, era esa la única decisión que privaba para terminar de conformar a los órganos legislativos del país.

Xihmai 74 
José Arturo Sosa Echeverría

El nuevo mecanismo para la integración de los congresos con diputados

de representación proporcional, una fórmula más democrática

Revista Xihmai XIII (26), 51-78, julio-diciembre 2018

Recientemente, ese esquema de configuración ha iniciado un cambio para incluir una fórmula eminentemente democrática, como se observa en el cuerpo del presente texto.

¿En qué consiste el cambio? En que algunas legislaciones electorales estatales han optado por conformar la representación proporcional con fórmulas de candidatos a la usanza tradicional, es decir, a través de listas inscritas solo para esa forma de elección provenientes de las dirigencias partidistas; asimismo, con fórmulas de candidatos que contendieron en la elección de mayoría relativa sin que el voto ciudadano les hubiera favorecido mayoritariamente.

En efecto, como hemos podido observar en este texto, algunas legislaciones estatales permiten, en la actualidad, la integración de las diputaciones locales electas bajo el principio de representación proporcional a través de dos mecanismos: El primero y original desde que se gestó la inclusión de este principio en el sistema electoral mexicano (1977) a través de una lista de fórmulas de candidatos presentada por los partidos políticos, lista que se ordena numéricamente para que, desde la primera posición, se vayan asignando las diputaciones al congreso respectivo. El segundo, reciente y novedoso mecanismo, que se presenta para quienes fueron registrados como candidatos en la elección de mayoría relativa y realizaron una campaña electoral, que invirtieron tiempo, trabajo, esfuerzo y dedicación para la obtención del voto ciudadano, pero que no lograron la mayoría de los votos en su distrito, pueden ahora ver recompensada su labor de campaña y su aceptación ciudadana con su incorporación en el órgano legislativo al tener la posibilidad de incluirles en una segunda lista de fórmulas de candidatos a integrar la legislatura de su entidad federativa.

Del análisis que realicé a las constituciones y legislaciones electorales del país, encontré que diez entidades federativas contienen en su marco normativo electoral los nuevos mecanismos de integración de sus respectivos poderes legislativos desde la perspectiva de la representación proporcional; no obstante, no todas esas legislaciones los tienen como una disposición imperativa, lo que hace que, en apariencia, se vislumbre como una disposición nueva y democrática, sin embargo, en la práctica se advierte que no se llega a concretar de tal forma.

Las entidades que incluyen en sus disposiciones jurídicas electorales la posibilidad de un doble mecanismo para arribar a la conformación de la legislatura bajo el principio de la representación proporcional pero que no lo establecen de un modo categórico, esto es, de forma tal que la asunción a las diputaciones por tal principio sea obligatoria y equilibrada, son: Guanajuato, Nayarit y Oaxaca. 
José Arturo Sosa Echeverría

El nuevo mecanismo para la integración de los congresos con diputados de representación proporcional, una fórmula más democrática

Revista Xihmai XIII (26), 51-78, julio-diciembre 2018

En el caso del estado de Guanajuato, no obstante que sus disposiciones legales atinentes sostienen el mecanismo de construcción del legislativo local de los candidatos provenientes de la representación proporcional a través de la doble vía (lista de partido para la representación proporcional y lista de candidatos que contendieron en la elección de mayoría relativa y que no obtuvieron el triunfo) y que obligan al organismo público local electoral a realizar la asignación de forma alternada entre ambas listas, la designación de los candidatos la harán a partir de las tres primeras fórmulas en la lista presentada por el partido para dicha contienda, y después, si es que algún partido tuviera la posibilidad de una cuarta asignación (lo que se antoja muy difícil, habida cuenta que las curules a repartirse por este principio son en un total de catorce, entre cuando menos nueve partidos nacionales contendientes), provendría de la segunda lista, es decir, la de candidatos que contendieron en la elección de mayoría relativa. En esas condiciones, no obstante que se advierte que este estado pudiera sumarse a la nueva forma de asignación, en la práctica no se llega a concretar este aspecto democrático.

Por lo que hace a los estados de Nayarit y Oaxaca, las legislaciones electorales correspondientes dejan abierta la posibilidad a los partidos políticos para que ellos determinen: en el primer caso, si llegan a incluirse a los candidatos que provienen de la contienda de mayoría relativa para efectos de realizar la asignación e ir asignándolos alternadamente entre las dos opciones; en el segundo, de decidir si las curules obtenidas bajo el sistema de la representación proporcional se asignarían, en su totalidad, de la lista que los propios institutos políticos presenten para tal efecto o que provengan de los candidatos registrados para la elección bajo el principio de mayoría relativa.

En los dos casos mencionados en el párrafo anterior, al no indicarse en forma terminante en sus disposiciones electorales, que la asignación deba efectuarse obligadamente de las dos listas de manera alternada, concluyo que, aunque en la ley se vislumbre un ánimo democrático, no se llega a concretar en razón de que dejan a los partidos políticos la decisión de la inclusión o no de los candidatos que provienen de las fórmulas que contendieron en la elección de mayoría relativa.

Respecto de los casos Aguascalientes, Baja California, Chihuahua, Ciudad de México, Hidalgo, Estado de México y Yucatán, son estados de la República que su marco normativo, constitucional y/o legal, sí establece de manera categórica e imperativa que en la asignación de las curules que arriban al cargo bajo el principio de la representación proporcional se lleve a cabo alternando las candidaturas entre dos opciones diferenciadas, esto es, entre una lista registrada por los partidos políticos para la asignación de las diputaciones plurinominales y la otra lista, la que proviene de las fórmulas registradas por los mismos partidos

Xihmai 76 
José Arturo Sosa Echeverría

El nuevo mecanismo para la integración de los congresos con diputados de representación proporcional, una fórmula más democrática

Revista Xihmai XIII (26), 51-78, julio-diciembre 2018

políticos que contendieron en la elección por el principio de mayoría relativa, cuando estas opciones no hayan logrado el triunfo en dicha elección.

El mecanismo adoptado por esas siete entidades federativas implanta una forma más democrática en la integración de las diputaciones locales, da a la representación proporcional un sentido legitimador en sus integrantes porque son ellos a quienes la ciudadanía otorgó su voto de manera directa.

El elector, al marcar la boleta a favor del candidato de su preferencia en la elección de diputados, nos está indicando que esa es la opción que pretende que le represente en la Cámara de Diputados; si esa candidatura no se ve favorecida por el voto mayoritario en el distrito correspondiente, pero logró un porcentaje significativo de votos, esa porción del electorado apreciará que sus sufragios tuvieron efectividad, que el objetivo intrínseco en la emisión de su voto se alcanza, lo que entonces legitima la asunción de esos candidatos a la cámara. Contrario Sensu, el elector no marca su boleta por la lista de candidatos de representación proporcional, podría decirse que por ellos no votan de manera directa, sin embargo llegan a la cámara, no hay relación directa entre el sufragio y el personaje electo.

Con la implementación de este nuevo mecanismo para la conformación de los órganos legislativos en el país, estamos avanzando en la consolidación de nuestra democracia, estamos dando mayor significado al voto activo, estamos logrando que el ciudadano aprecie que su voto, aunque no formó parte de una mayoría relativa, es considerado en la integración de la Cámara de Diputados, lo que en su caso pudiera favorecer los índices de participación electoral.

Me parece importante incorporar en todas las disposiciones electorales del país los mecanismos adoptados por los estados de la República mencionados, creo que la inclusión de estos métodos novedosos aportan mucho a la democracia participativa de México.

\section{Fuentes de Consulta}

BORJA, R. Enciclopedia de la Política. Recuperado el 9 de octubre de 2018 de www.enciclopediadelapolitica.org

Constituciones Políticas y legislaciones electorales de las siguientes entidades federativas:

Aguascalientes (2015). Periódico Oficial del Estado. 
José Arturo Sosa Echeverría

El nuevo mecanismo para la integración de los congresos con diputados de representación proporcional, una fórmula más democrática Revista Xihmai XIII (26), 51-78, julio-diciembre 2018

Baja California (2015 y 1953). Periódico Oficial del Estado.

Ciudad de México (2017). Periódico Oficial.

Guanajuato (2014). Periódico Oficial del Estado.

Hidalgo (2014). Periódico Oficial del Estado.

Estado de México (2014). Periódico Oficial del Estado.

Nayarit (2010). Periódico Oficial del Estado.

Oaxaca (2017). Periódico Oficial del Estado.

Yucatán (2014). Periódico Oficial del Estado.

Ley General de Instituciones y Procedimientos Electorales. Diario Oficial de la Federación (2014). México.

PÉREZ PORTO, J. y A. Gardey. Cociente.

REAL ACADEMIA ESPAÑOLA (2017). Diccionario de la lengua española. Madrid. 